\title{
Magnetic Properties of Helicobacter Pylori Ferritins Genetically Prepared Under Different Biomineralization Conditions
}

\author{
K. Son ${ }^{1}$, J.-N. Park ${ }^{1}$, Sungwon Yoon ${ }^{2}$, B. J. Suh ${ }^{2}$, K. J. Cho ${ }^{3}$, K. H. Kim ${ }^{4}$, and Z. H. Jang ${ }^{\text {* }}$ \\ ${ }^{1}$ Department of Physics, Kookmin University, Seoul 02707, Korea \\ ${ }^{2}$ Department of Physics, The Catholic University of Korea, Bucheon 14662, Korea \\ ${ }^{3}$ Department of Life Sciences \& Biotechnology, Korea University, Seoul 02841, Korea \\ ${ }^{4}$ Department of Biotechnology \& Bioinformatics, Korea University, Sejong 30019, Korea
}

(Received 20 December 2015, Received in final form 10 January 2016, Accepted 11 January 2016)

\begin{abstract}
Magnetic properties of bio-magnetic molecule ferritin have been investigated. Two ferritin samples were synthesized under different magnetic fields, 0 and $9.4 \mathrm{~T}$, respectively. This work is focused on the influence of magnetic field on biomineralization process. While magnetization vs. temperature (M-T) data of both samples measured at 1000 Oe are almost identical except for low temperature region $(\mathrm{T}<6 \mathrm{~K})$, magnetization vs. field (M-H) data show noticeable difference. From an analysis of M-H data by using a modified Langevin function, we could extract the saturation magnetization $m_{0}(T)$, the effective magnetic moment $\mu_{\text {eff }}(T)$ and the linear susceptibility $\chi(T)$. The difference between the samples is most prominent in the $\chi(T)$, whereby the $\chi(T)$ of the sample prepared at $9.4 \mathrm{~T}$ is 1.7 times bigger than that of the other. In addition, from hysteresis and relaxation measurements, we found the sample prepared at $9.4 \mathrm{~T}$ showed strikingly smaller coercivity and slower relaxation.
\end{abstract}

Keywords : magnetic nanoparticle, ferritin, biomineralization

\section{Introduction}

Magnetic nanoparticle systems are an important research topic for various future applications including magnetic data storage [1], hyperthermia treatment agent [2], etc. Also, finite-size effect study and surface effect study can be performed with magnetic nanoparticle systems as model systems. Thus there have been many research reports on various different types of magnetic nanoparticle systems.

Among the various nanoparticle systems, ferritin has long been studied with many interesting results with its peculiar properties. Ferritin is composed of protein shell and magnetic mineral core. The outer diameter of protein shell is about $\sim 12 \mathrm{~nm}$ [3] and inner diameter of the hollow space in the protein shell is about $8 \mathrm{~nm}$. The protein shell has channels through which mineral ions pass and solidify in the hollow space [4]. The mineral magnetic core is roughly known to have chemical composition of iron oxide hydroxide ferrihydrite with nominal

(C)The Korean Magnetics Society. All rights reserved.

*Corresponding author: Tel: +82-2-910-4768

Fax: +82-2-910-4728, e-mail: zeehoonj@kookmin.ac.kr formula, $\left(\mathrm{FeOOH} \cdot n \mathrm{H}_{2} \mathrm{O}\right)$ and the nominal diameter is known to be around $5 \mathrm{~nm}$ [5]. The ferritin core is known to have internal antiferromagnetic order but the uncompensated spins due to the truncated lattice at the surface and the breakage of antiferromagnetic order at the internal defects cause superparamagnetic behavior [3-5]. Ferritin is an interesting model system for the study of magnetism in nanometer scale because of its rather regular size of magnetic core and the thick protein shell which almost nullifies inter-particle interaction. The magnetic properties and related phenomena in ferritin have been studied by many researchers. But complete understanding is not attained yet.

Biomineralization is the process that mineral ions pass through the protein shell and solidify in the inner empty space of the protein structure [4]. The biomineralization process is an important fundamental issue in biology especially for the storage of iron in non-toxic form but our knowledge about the process is very little. It is quite a complex process that there are lots of open questions including how the magnetic field affects the whole process and the magnetic properties of final mineral core.

Direct observation of the biomineralization process is 
quite a difficult work to be done because of lack of appropriate experimental techniques and limitations in experimental tools. Our motivation of this research is to study an aspect of the biomineralization by investigating the magnetic properties of ferritin samples with different biomineralization conditions.

\section{Sample}

Ferritin gene was obtained from $H$. pylori and expressed from a plasmid in E.coli expression system and the apoferritins (protein shells of ferritin) were placed under different magnetic field conditions. Reconstitution process for sample 1 was performed under normal condition of earth's magnetic field. Magnetic field of 94000 Oe was applied during the iron intake process of sample 2. The reconstitution processes took about 30 minutes for both samples. Considering reconstitution rate and time, we conjectured that both samples contain about 4000 iron ions per ferritin in average.

\section{Experiment}

Conventional SQUID (Superconducting Quantum Interference Device) magnetometer was extensively utilized to measure magnetization of the samples. Temperature dependent magnetization was measured from $2 \mathrm{~K}$ to 300 $\mathrm{K}$ at the external magnetic field of 1000 Oe with ZeroField-Cool (ZFC) protocol and Field-Cool (FC) protocol. Field dependence of magnetization was measured at various temperatures between $2 \mathrm{~K}$ and $200 \mathrm{~K}$. The hysteresis curves of magnetization were also measured at $2 \mathrm{~K}$. After cooling the samples down to $2 \mathrm{~K}$ with external magnetic field of $100 \mathrm{Oe}$, the magnetization relaxation curves were obtained by measuring magnetization of the samples continuously as a function of time after turning off the magnet.

\section{Results and Discussion}

Magnetization vs. temperature (M-T) data (See Fig. 1) of both samples show typical superparamagnetic behavior. The bifurcation temperature between the FC data and ZFC data for both samples are almost identical to be $\sim 13$ $\mathrm{K}$ indicating the maximum blocking temperatures of both samples are almost the same. The FC magnetization data for both samples are almost identical down to $8 \mathrm{~K}$ but the discrepancy increases as the temperature is lowered further down. For both samples, ZFC data show broad maximum around $8 \mathrm{~K}$ indicating the distribution of the anisotropy barrier in the samples. The difference between
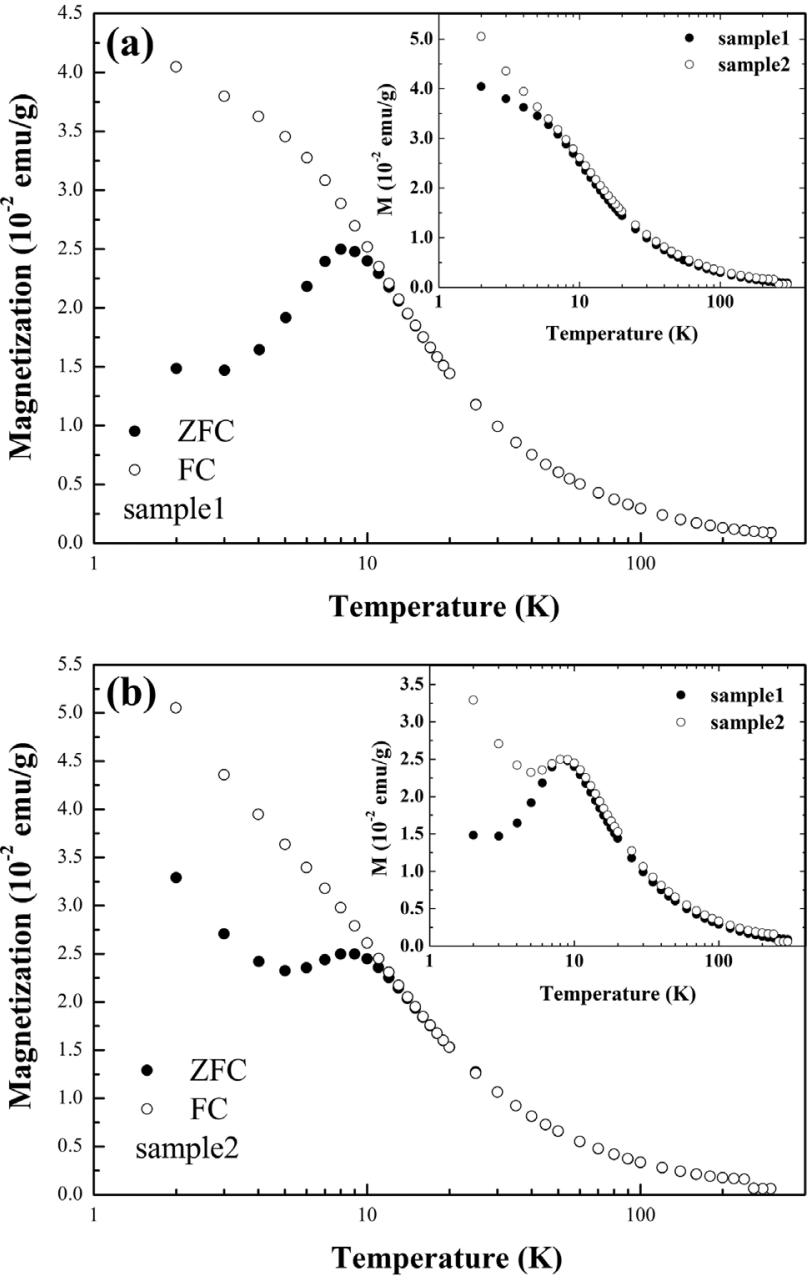

Fig. 1. Temperature dependence of magnetization in ZFC and FC process. (a) Temperature dependence of magnetization of sample 1. Inset: Comparison of FC curves between two samples. (b) Temperature dependence of magnetization of sample 2. Inset: Comparison of ZFC curves between the samples.

the two ZFC data sets is that the data for sample 2 show rather large upshot at low temperature suggesting some portion of the sample is paramagnetic. The data for sample 1 also shows the paramagnetic behavior at low temperature but the amount of the paramagnetic upshot is less than that of sample 2 .

It is noted that both samples are prepared in the same batch and only difference is the environment (magnetic environment) of the biomineralization. Besides the paramagnetic behavior, the influence of the magnetic field in the biomineralization process was not noticeable in the temperature dependence of the magnetization of those ferritin samples, at least, at low field.

Field dependence of magnetization was measured at various temperatures from $2 \mathrm{~K}$ up to $200 \mathrm{~K}$ and the selected data sets are shown in Fig. 2. As is evident in the 


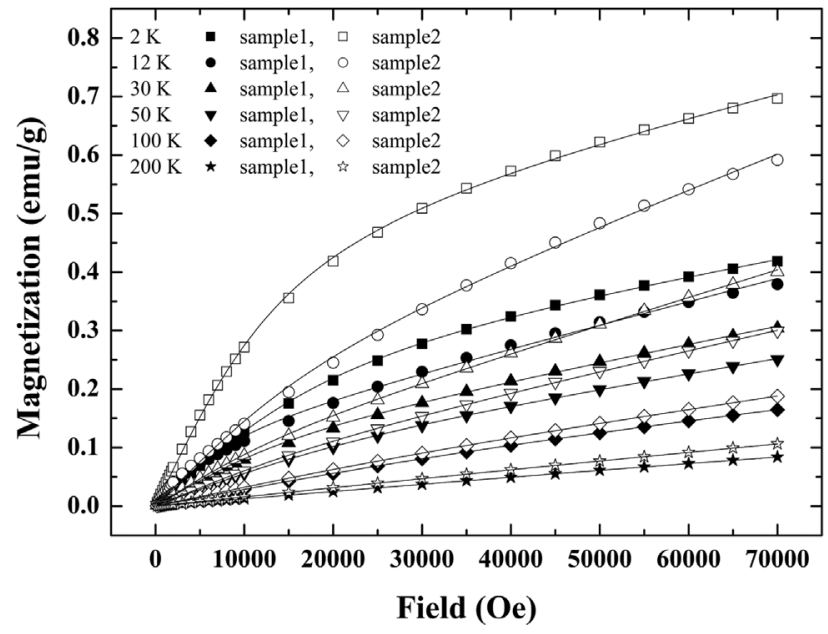

Fig. 2. Field dependence of magnetization measured at various temperatures. Solid lines are fitting results of the corresponding data with modified Langevin function.

data, the magnetization of sample 2 is bigger than that of sample 1. For example, at $2 \mathrm{~K}$ and at $70000 \mathrm{Oe}$, the magnetization of sample 2 is 1.65 times bigger than that of sample 1 and the trend is quite general for all the other temperature and field conditions of measurements. Usual $M-H$ data of ferritin cannot be analyzed with simple Langevin function since it has linearly increasing nonsaturating behavior as the field is increased. Thus we analyzed the $M-H$ data by fitting with a modified Langevin function [6]. The modified Langevin function has additional term which is linear in field and is expressed as,

$$
M(H, T)=m_{0}(T)\left[\operatorname{coth}\left(\frac{\mu_{e f f}(T) H}{k_{B} T}\right)-\left(\frac{k_{B} T}{\mu_{\text {eff }}(T) H}\right)\right]+\chi H
$$

where $m_{0}(T)$ is saturation magnetization, $\mu_{\text {eff }}$ is temperature dependent effective magnetic moment of the particles, $k_{B}$ is Boltzmann constant, $H$ is external magnetic field, $T$ is the temperature of the sample and $\chi(T)$ is additional temperature dependent susceptibility. The quality of fitting is quite good as is shown in Fig. 2 implying that modified Langevin function is a good analysis model for ferritin samples.

From the fitting, we could obtain saturation magnetization, $m_{0}(T)$, effective moment, $\mu_{e f f}$, and linear susceptibility, $\chi(T)$, as functions of temperature and the data are shown in Fig. 3.

For both samples, effective magnetic moment $\mu_{e f f}$ shows broad maximum around $150 \mathrm{~K}$ in the temperature range from $30 \mathrm{~K}$ to $200 \mathrm{~K}$. The magnetic core of ferritin is known to have internal antiferromagnetic order at low temperature and the effective magnetic moment arises due
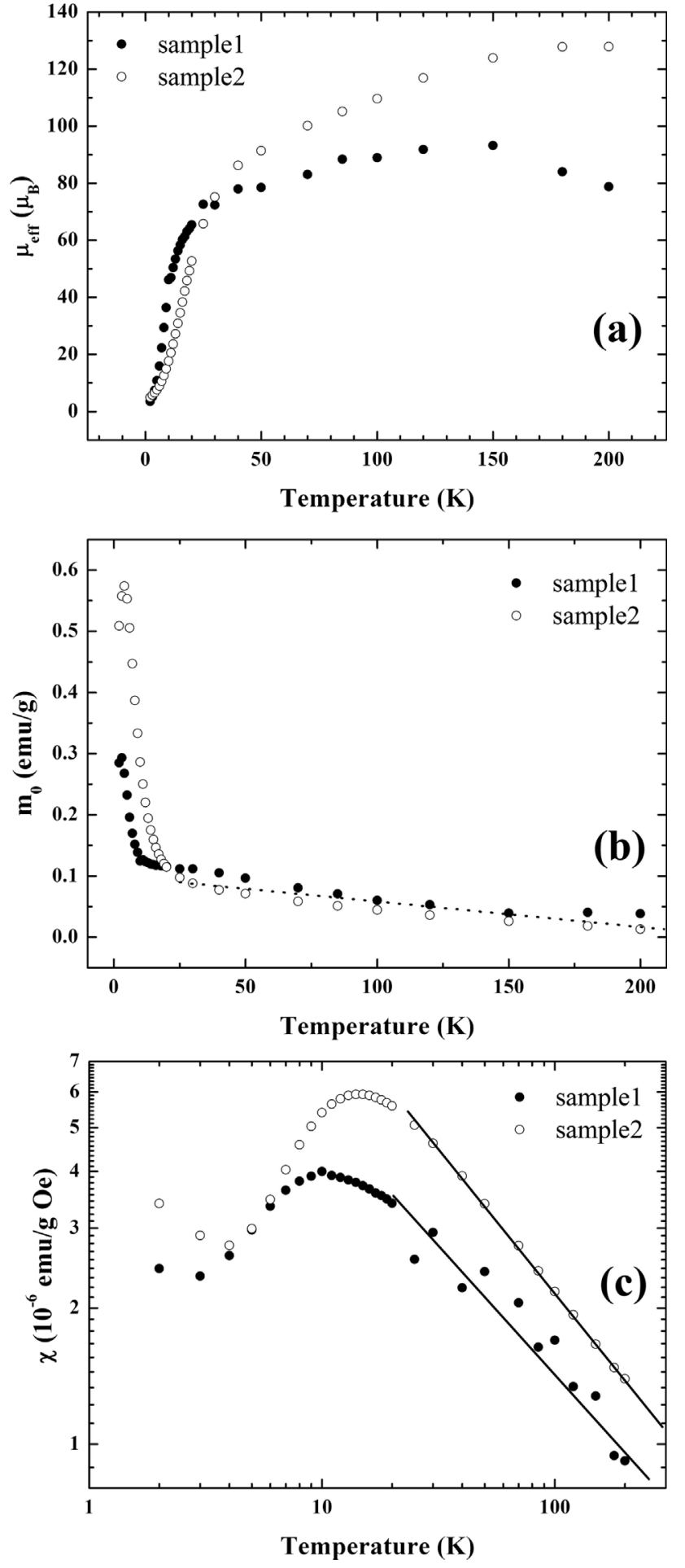

Fig. 3. Temperature dependence of the parameters deduced from fitting $M-H$ data with modifield Langevin function (a) Temperature dependence of effective moment, $\mu_{\text {eff }}$ (b) Temperature dependence of saturation magnetization, $m_{0}$. Dotted line is fitting curve with $m_{0}=m_{*}\left(T_{N}-T\right) / T_{N}$. (c) Temperature dependence of linear susceptibility, $\chi(T)$. Solid line is a guide to eye and designates the paramagnetic behavior $(\chi(T) \propto 1 / T)$ of the linear susceptibility at high temperature. 
to the incompletion of the antiferromagnetic order at the boundary and defects. We have tried to fit the $\mu_{\text {eff }}$ data with bulk antiferromagnetic magnon law [5], $\mu(0)\left(1-\alpha T^{2}\right)$, but the fitting was not possible. There may be a possibility of lacking crystallinity in the magnetic core. Mammalian ferritin cores are usually more crystalline in structure but ferritins from viruses or other bacterium have cores which are more amorphous-like in structure [7]. Also, usual mammalian ferritin like horse spleen ferritin has magnetic core which is a mixture of crystalline particles and some amorphous residue of mineralization process [7]. Thus one can conjecture that H. Pylori ferritin may not have complete crystalline structured core.

Below $30 \mathrm{~K}, \mu_{\text {eff }}$ decreases rapidly as the temperature is lowered. The fast reduction of the effective moment can be due to the intra-particle antiferromagnetic interaction in the magnetic core of ferritin. Sample 2 has bigger effective moment in high temperature range but the decrease of the effective moment at low temperature is faster in sample 1.

The temperature dependence of the saturation magnetization $m_{0}(T)$ in both samples is very similar, showing steep rise at low temperature and slow increase at high temperature as the temperature is lowered. But the saturation magnetization of sample 2 is eminently higher at low temperatures and, above $25 \mathrm{~K}$, both samples have very similar saturation magnetization behavior. By using an empirical temperature dependence of saturation magnetization, $m_{0}(T)=m_{*}\left(T_{N}-T\right) / T_{N}$, where $T_{N}$ is Néel temperature and the value of saturation magnetization extrapolated to $0 \mathrm{~K}$ is denoted as $m_{*}[6,8,9]$. The saturation magnetization of both samples show roughly linear temperature dependence above $25 \mathrm{~K}$ and Néel temperature was found to be $240 \mathrm{~K}$ which is quite a good agreement with previous report with Mössbauer study [10].

The linear susceptibility is suggested to be due to the progressive rotation of the sub-lattice magnetization in the antiferromagnetic nanoparticle as the field is increased [6] whereas the uncompensated spin at the surface of the nanoparticle and the uncompensated spin due to the internal defects give rise to the superparamagnetic behavior. Susceptibility $\chi(T)$ shows enormous difference between the two samples. From $\sim 15 \mathrm{~K}$ up to $200 \mathrm{~K}$, the $\chi(T)$ of sample 2 is almost 1.7 times bigger than that of sample 2 and $\chi(T)$ of both samples show typical paramagnetic behavior with the inverse temperature dependence. Below $\sim 15 \mathrm{~K}, \chi(T)$ of both samples show complex behavior such that $\chi(T)$ decreases below $\sim 15 \mathrm{~K}$ with small upturn at very low temperature region. The complex behavior cannot be explained with usual paramagnetic behavior and it may be due to the interparticle interaction.

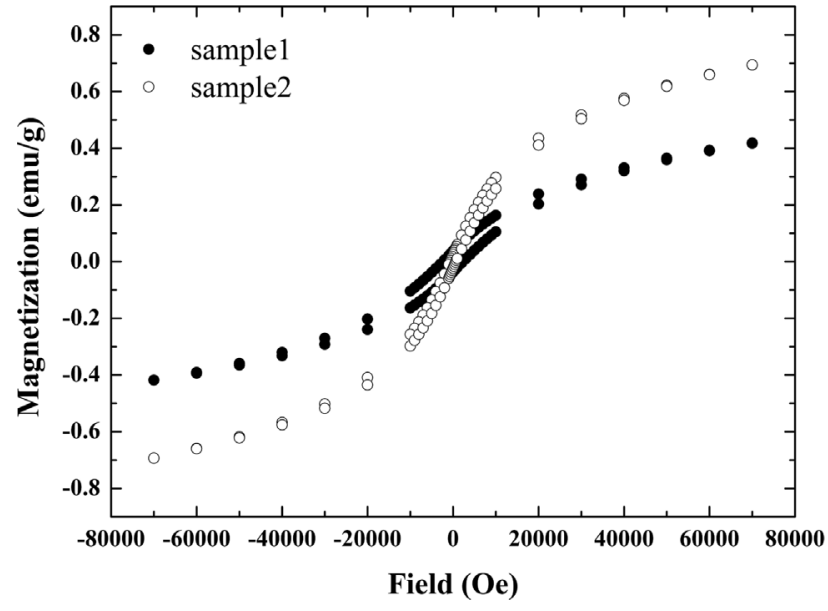

Fig. 4. Hysteresis curves of both samples measured at $2 \mathrm{~K}$. The hysteresis curves show most striking contrast between the samples.

Among all the magnetization measurements, hysteresis measured at $2 \mathrm{~K}$ shows most striking differences between the two samples. Coercive field of sample 1 is 2400 Oe but the coercive field of sample 2 is 700 Oe about 3.5 times smaller than that of sample 1. But the magnetization at 70000 Oe is bigger in sample 2 as shown in Fig. 4. This opposite behavior in hysteresis curves may be understood with the difference in paramagnetic contents in both samples : The crossing point between the hysteresis and $\mathrm{x}$-axis moves up(down) in the first(third) quadrant when linear paramagnetic component is added. Thus coercive field is reduced. Also, the addition of the linear paramagnetic component increases high field magnetization since paramagnetic magnetization is along the direction of the magnetic field. To check whether the difference between the hysteresis curves is due to the paramagnetic component, we have fitted the difference between the hysteresis curves of two samples with Langevin function. But the extracted paramagnetic component described with the Langevin function cannot explain the difference between the two ZFC data sets. Thus we could not conclude that the difference in the magnetization is simply due to the difference in paramagnetic component in the samples.

Relaxation of magnetization at $2 \mathrm{~K}$ was measured for both samples prepared in field-cool condition and the results are shown in Fig. 5. Both samples show linear relaxation behavior (linear dependence of magnetization on $\ln (t)$ after $500 \mathrm{~s})$. We have fitted the linear section of relaxation with $M(t)=M_{0}-S \ln \left(t / t_{0}\right)$, where $t_{0}$ is reference time, $t$ is elapsed time after magnet turn-off, $M_{0}$ is the magnetization at $t_{0}, M(t)$ is magnetization at time $t$ and $S$ is magnetic viscosity [11]. We found that the values of 


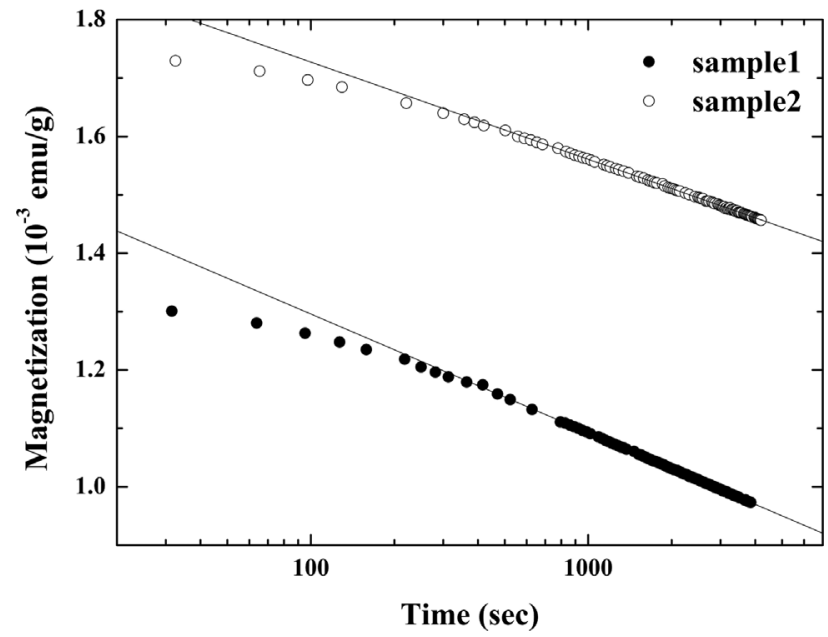

Fig. 5. Magnetization relaxation measured at $2 \mathrm{~K}$ just after turning off the magnetic field. Solid lines are fitting curves with the equation described in the text to find the magnetic viscosity, $S$.

viscosity, $S$, which is defined as $S \equiv \partial M / \partial(\ln t)$, are 8.84 $\times 10^{-5}(\mathrm{emu} / \mathrm{g})$ for sample 1 and $7.23 \times 10^{-5}(\mathrm{emu} / \mathrm{g})$ for sample 2. It is noted that the relaxation after $500 \mathrm{~s}$ is faster in sample 1 than in sample 2 as is obvious from the higher value of viscosity in sample 1 . The behavior is quite interesting if one considers almost identical average blocking temperature of both samples and blocking temperature distribution (which can be inferred from the ZFC and FC data.).

\section{Summary}

We have studied magnetism of two ferritin samples prepared under different magnetic field conditions $(0 \mathrm{Oe}$ and 94000 Oe). Low field temperature dependent magnetization data of both samples are almost identical to each other except low temperature paramagnet-like behavior. Field dependent magnetization measured at various temperatures from $2 \mathrm{~K}$ to $200 \mathrm{~K}$ and from 0 Oe up to 70000 Oe could be fitted well with the modified Langevin function and effective magnetic moment, saturation magnetization, and temperature dependent linear susceptibility have been deduced from the fitting. Effective magnetic moment, saturation magnetization, linear susceptibility, all showed difference between the samples and, especially, the difference in the linear susceptibility is largest among them. Overall, the magnetization of the sample prepared under magnetic field is bigger in field dependent magnetization data. Hysteresis data measured at $2 \mathrm{~K}$ showed the most striking difference between the samples: The sample prepared under magnetic field showed smaller coercivity but showed bigger magnetization at 70000 Oe. Relaxation of the magnetization also showed difference such that the sample prepared at zero external field has smaller relaxation rate.

It is noted that not much research has been performed on the influence of the magnetic field on biomineralization process and the underlying mechanism in the biomenralization not well understood yet. Even though we have observed differences in the magnetic properties of ferritin samples prepared under different magnetic environment, our observation may not be final and more advanced and detailed research on the same topic is under way.

\section{References}

[1] A. Ethirajan, U. Wiedwald, H.-G. Boyen, B. Kern, L. Han, A. Klimmer, F. Weigl, G. Kästle, P. Ziemann, K. Fauth, J. Cai, R. Jürgen Behm, A. Romanyuk, P. Oelhafen, P. Walther, J. Biskupek, and U. Kaiser, Adv. Mate. 19, 406 (2007).

[2] A. Jordan, R. Scholz, P. Wust, H. Fahling, and R. Felix, J. Magn. Magn. Mater. 210, 413 (1999).

[3] C. Gilles, P. Bonville, H. Rakoto, J. M. Broto, K. K. W. Wong, and S. Mann, J. Magn. Magn. Mater. 241, 430 (2002).

[4] N. D. Chasteen and P. M. Harrison, J. Struct. Biol. 120, 182 (1999).

[5] N. J. O. Silva, V. S. Amaral, and L. D. Carlos, Phys. Rev. B 71, 184408 (2005).

[6] S. A. Makhlouf, F. T. Parker, and A. E. Berkowitz, Phys. Rev. B 55, 14717 (1997).

[7] William H. Massover, Micron 24, 389 (1993).

[8] M. S. Seehra and A. Punnoose, Phys. Rev. B 64, 132410 (2001).

[9] M. S. Seehra, V. S. Babu, A. Manivannan, and J. W. Lynn, Phys. Rev. B 61, 3513 (2000).

[10] E. R. Bauminger and I. Nowik, Hyperfine interactions 50, 489 (1989).

[11] M. M. Ibrahim, S. Darwish, and M. S. Seehra, Phys. Rev. B 51, 2955 (1995). 